University of Wollongong

Research Online

Faculty of Informatics - Papers (Archive)

Faculty of Engineering and Information

Sciences

1987

\title{
A construction for orthogonal designs with three variables
}

Jennifer Seberry

University of Wollongong, jennie@uow.edu.au

Follow this and additional works at: https://ro.uow.edu.au/infopapers

Part of the Physical Sciences and Mathematics Commons

\section{Recommended Citation}

Seberry, Jennifer: A construction for orthogonal designs with three variables 1987.

https://ro.uow.edu.au/infopapers/1029

Research Online is the open access institutional repository for the University of Wollongong. For further information contact the UOW Library: research-pubs@uow.edu.au 


\title{
A construction for orthogonal designs with three variables
}

\author{
Abstract \\ We show how orthogonal designs $O D\left(48 p^{2} t ; 16 p^{2} t, 16 p^{2} t, 16 p^{2} t\right)$ can be constructed from an Hadamard \\ matrix of order $4 p$ and an $\mathrm{OD}(4 \mathrm{t}, \mathrm{t}, \mathrm{t}, \mathrm{t}, \mathrm{t})$. This allows us to assert that $\mathrm{OD}\left(48 \mathrm{p}^{2} \mathrm{t} ; 16 \mathrm{p}^{2} \mathrm{t}, 16 \mathrm{p}^{2} \mathrm{t}, 16 \mathrm{p}^{2} \mathrm{t}\right)$ exist for \\ all $t, p \leq 102$ except possibly for $t \in\{67,71,73,77,79,83,86,89,91,97\}$. These designs are new. \\ Disciplines \\ Physical Sciences and Mathematics \\ Publication Details \\ Seberry, J, A construction for orthogonal designs with three variables, Combinatorial Design Theory, (C.J. \\ Colbourne and R.A. Mathon, (Eds)), North Holland, 1987, 437-440.
}




\title{
A Construction for Orthogonal Designs with Three Variables
}

\author{
Jennifer Seberry
}

Department of Computer Science University College

The University of New South Wales

Australian Defence Forces Academy

Canberra, A.C.T. 2600

AUSTRALIA

TO ALEX ROSA ON HIS FIFTIETH BIRTHDAY

\section{ABSTRACT}

We show how orthogonal designs $O D\left(48 p^{2} t ; 16 p^{2} t, 16 p^{2} t, 16 p^{2} t\right)$ can be construeted from an Hadamard matrix of order $4 p$ and an $O D(4 t ; t, t, t, t)$. This allows us to assert that $O D\left(48 p^{2} t\right.$; $\left.16 p^{2} t, 16 p^{2} t, 16 p^{2} t\right)$ exist for all $t, p \leq 102$ except possibly for $t \in\{67,71,73,77,79,83,86,89,91,97\}$. These designs are new.

\section{Introduction}

Let $H=\left(h_{i j}\right)$ be a matrix of order $h$ with $h_{i j} \in\{1,-1\} . H$ is called an Hadamard matrix of order $n$, if $H H^{T}=h I_{h}$ where $I_{h}$ denotes the identity matrix order of $h$.

An orthogonal design $A$, of order $n$, type $\left(p_{1}, p_{2}, \ldots, p_{4}\right)$, denoted $O D\left(n ; p_{1}, p_{2}, \ldots, p_{u}\right)$, on the commuting variables $\left( \pm x_{1}, \pm x_{2}, \ldots, \pm x_{u}, 0\right)$ is a square matrix of order $n$ with entries $\pm x_{k}$ where each $x_{k}$ occur $p_{k}$ times in each row and column such that the rows are pairwise orthogonal.

In other words

$$
A A^{T}=\left(p_{1} x_{1}^{2}+\cdots+p_{u} x_{u}^{2}\right) I_{n} .
$$

It is known that the maximum number of variables is an orthogonal design is $\rho(n)$, the Radon number, where for $n=2^{a} b, b$ odd, set $a=4 c+d, 0 \leq d<4$, then $\rho(n)=8 c+2^{d}$.

$O D(4 t ; t, t, t, t)$, otherwise called Baumert-Hall arrays, and $O D\left(2^{p} ; a, b, 2^{p}-a-b\right)$ have been extensively used to construct Hadamard matrices and weighing matrices. For details see Geramita and Seberry (1979). 
Geramita, Geramita and Wallis (-Seberry) observed (see Geramita and Seberry $[1979, \S 4.3])$ that if $A, B, C, D$ are four eirculant or type 1 matrices of order $n$ satisfying

$$
A A^{T}+B B^{T}+C C^{T}+D D^{T}=\left(\sum_{i=1}^{u} p_{i} x_{i}^{2}\right) I_{n}
$$

then $A, B, C, D$ can be used in the Goethals-Seidel array or (J. Seberry WallisWhiteman array)

$$
\left[\begin{array}{cccc}
A & B R & C R & -D R \\
-B R & A & D^{T} R & -C^{T} R \\
C R & -D^{T} R & A & B^{T} R \\
D R & C^{T} R & -B^{T} R & A
\end{array}\right]
$$

to form an orthogonal design $O D\left(4 n ; p_{1}, p_{2}, \ldots, p_{u}\right)$.

\section{Background}

Kharaghani (1985) defined $C_{k}=\left[h_{i j}, h_{k j}\right]$ and applying that to Hadamard matrices of order $4 p$, obtained matrices satisfying

$$
\begin{gathered}
C_{i} C_{j}=0, i \neq j \\
\sum_{i=1}^{4 p} C_{i}^{2}=(4 p)^{2} I_{4 p} .
\end{gathered}
$$

He then used this to show there are Bush-type (blocks $J_{4 p}$ down the diagonal) and Szekeres-type ( $h_{i j}=-1 \Rightarrow h_{j i}=1$ and not necessarily vice versa) Hadamard matrices. By using a symmetric Latin square he could also have shown that regular symmetric Hadamard matrices with constant diagonal of order $(4 p)^{2}$ could be constructed by his method.

Hammer, Sarvate and Seberry applied Kharaghani's method to $O D\left(n ; s_{1}, \ldots, s_{y}\right)$ and in particular $O D(4 t ; t, t, t, t)$ and $O D 4 s ; s, s, s, s)$ obtaining existence of $O D\left(48 s^{2} t\right.$ $\left.12 s^{2} t, 12 s^{2} t, 12 s^{2} t, 12 s^{2} t\right)$ and $O D\left(80 s^{2} t ; 20 s^{2} t, 20 s^{2} t, 20 s^{2} t, 20 s^{2} t\right)$ from $O D(4 s ; s, s, s, s)$ and $O D(4 t ; t, t, t, t)$. Seberry (to appear) extended this further to obtain $O D\left(16 k s^{2} t ; 4 k s^{2} t, 4 k s^{2} t, 4 k s^{2} t, 4 k s^{2} t\right)$ for $\{1,3,5, \ldots\}$.

We modify their techniques to obtain new orthogonal designs.

\section{Construction}

Let $C_{1}, C_{2}, \ldots, C_{4 p}$ be the Kharaghani matrices of order $4 p$ obtained from an orthogonal design $O D(4 p ; p, p, p, p)$.

Let $a, b, c$ be commuting variables and write

$$
\begin{gathered}
{\left[a C_{1}: a C_{2}: \ldots: a C_{p}: b C_{p+1}: \ldots . b C_{2 p}: c C_{2 p+1}: \ldots: c C_{3 p}\right]} \\
{\left[a C_{3 p+1}: \ldots: a C_{4 p}: c C_{p+1}: \ldots .: c C_{2 p}: b C_{2 p+1}: \ldots .: b C_{3 p}\right]}
\end{gathered}
$$


for the first rows of two block circulant matrices $W_{1}$ and $W_{2}$. It can be checked that $W_{1} W_{2}^{T}=W_{2} W_{1}^{T}$. Write

$$
\begin{gathered}
{\left[a C_{2 p+1}: \ldots . .: a C_{3 p}: b C_{1}: \ldots: b C_{p}: c C_{3 p+1}: \ldots .: c C_{4 p}\right]} \\
{\left[a C_{p+1}: \ldots . a C_{2 p}: b C_{1}: \ldots b C_{p}: b C_{3 p+1}: \ldots b C_{4 p}\right]}
\end{gathered}
$$

for the first rows of two block back-circulant matrices $W_{3}$ and $W_{4}$. It can be checked that $W_{3} W_{4}^{T}=W_{4} W_{3}^{T}$.

Now by virtue of being circulant and back-circulant

$$
W_{i} W_{j}^{T}=W_{j} W_{i}^{T} \quad i \in\{1,2\}, j \in\{3,4\} .
$$

Example. Let $p=3$ so there are 12 matrices of order $12, C_{1}, \ldots, C_{12}$. Then

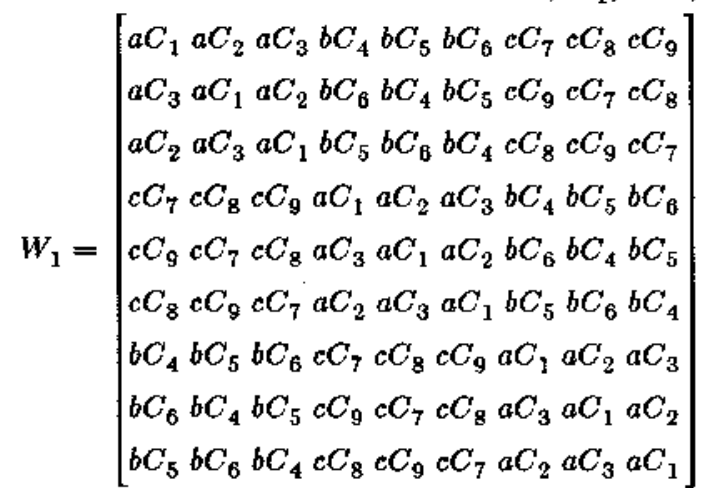

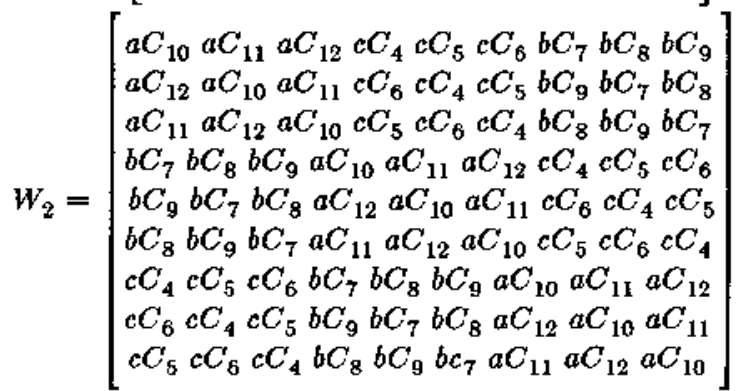

$$
\begin{aligned}
& W_{1} W_{2}^{T}=I_{q} \times b c \sum_{i=4}^{q} C_{i} c_{i}^{T}
\end{aligned}
$$

but $C_{i} C_{i}^{T}$ is symmetric and so $W_{1} W_{2}^{T}=W_{2} W_{1}^{T}$.

$W_{3}$ and $W_{4}$ are formed similarly but are back-circulant in blocks (i.e., type 2) in the language of Seberry-Wallis and Whiteman).

Thus $W_{1}, W_{2}, W_{3}, W_{4}$ are Witliamson-type matrices of order $12 p^{2}$ they can be used to replace the variables of an $O D(4 ; 1,1,1,1)$ to get an $O D\left(48 p^{2} ; 16 p^{2}, 16 p^{2}, 16 p^{2}\right.$, $\left.16 p^{2}\right)$. In general they can be used to replace the variables of an $O D(4 t ; t, t, t, t)$ so we have 
Theorem: If there is an Hadamard matrix of order $4 p$ and an $O D(4 t ; t, t, t, t)$ then there is an $O D\left(48 p^{2} t ; 16 p^{2} t, 16 p^{2} t, 16 p^{2} t, 16 p^{2} t\right)$.

Since Hadamard matrices of order $4 p$ exist for all $p \leq 102$ and $O D(4 t ; t, t, t, t)$ exist for all $t \leq 102$ except possibly for $t \in S, S=\{67,71,73, \overline{77}, 79,83,86,89,91,97\}$ (see Seberry (1986a)) we have

Corollary: $O D\left(48 p^{2} t ; 16 p^{2} t, 16 p^{2} t, 16 p^{2} t, 16 p^{2} t\right)$ exist for all $t, p \leq 102$ except possibly for $t \in S$.

\section{Acknowledgement}

The author's research is supported in part by grants from ACRB and ATERB.

\section{References}

[1] J. Cooper and J. Seberry Wallis, "A construction of Hadamard arrays", Bull. Austral Math. Soc., 7 (1972) 269-278.

[2] A.V. Geramita and J. Seberry, Orthogonal Designs: Quadratic Forms and Hadamard Matrices Marcel Dekker, New York-Basel (1986).

[3] J. Hammer, D.G. Sarvate, J. Seberry, "A note on orthogonal designs", Ars Combinatoria, submitted.

[4] H. Kharagani, "New class of weighing matrices", Ars Combinatorica, Vol. 19, pp. 69-72.

[5] J. Seberry, "More towards proving the Hadamard conjecture", (preprint).

[6] J. Seberry-Wallis, "Hadamard matrices, Part IV of W.D. Wallis, A. P. Street, and J. Seberry-Wallis", Combinatorics: Room Square, sum free sets and Hadamard matrices, Lecture Notes in Mathematics, Vol, 292, Springer-Verlag, BerlinHeidelberg New York. 